\title{
Fractures sociales et inégalités scolaires
}

Social breakdown and educational inequality

Fracturas sociales y desigualdades escolare

\section{Pierre-Louis Gauthier}

\section{OpenEdition}

\section{Journals}

Édition électronique

URL : https://journals.openedition.org/ries/745

DOI : $10.4000 /$ ries.745

ISSN : 2261-4265

\section{Éditeur}

France Education international

Édition imprimée

Date de publication : 1 décembre 2009

Pagination : 85-92

ISBN : 978-2-8542-0577-0

ISSN : 1254-4590

\section{Référence électronique}

Pierre-Louis Gauthier, «Fractures sociales et inégalités scolaires », Revue internationale d'éducation de Sèvres [En ligne], 52 | décembre 2009, mis en ligne le 01 décembre 2012, consulté le 25 avril 2022 URL : http://journals.openedition.org/ries/745 ; DOI : https://doi.org/10.4000/ries.745 


\section{Fractures sociales et inégalités scolaires}

\section{Pierre-Louis Gauthier}

L'ordre de la nature n'établit dans la société d'autre inégalité que celle de l'instruction et de la richesse; et, étendant l'instruction, vous affaiblirez à la fois les effets de ces deux causes de distinction.

Rapport du marquis de Condorcet (Assemblée législative, août 1792)

Issu du siècle des Lumières, le principe d'équité émerge en 1789. Conséquence de l'égalité, cette "passion française » selon Tocqueville, le droit naturel à l'éducation suivant le mérite et non plus la fortune ou la naissance, reçoit un début de concrétisation avec les projets révolutionnaires. Depuis, il n'a cessé d'inspirer les pays démocratiques qui ont tous inscrit ce principe premier sur le socle de leurs systèmes éducatifs, s'efforçant, en un laborieux cheminement, de l'appliquer à la réalité scolaire.

\section{ÉGALITÉ versus QUALITÉ SCOLAIRE ?}

Pourtant, force est de reconnaître, en ce $\mathrm{XXI}^{\mathrm{e}}$ siècle, alors que l'école reçoit de plein fouet les fortes inégalités sociales et culturelles de nos sociétés en mutation, que les inégalités scolaires taraudent les systèmes éducatifs, en dépit d'efforts plus que centenaires en faveur de l'équité. On peut même affirmer que l'un des traits communs à tous les systèmes est celui des inégalités. L'égalité devant l'éducation est-elle devenue une utopie ? L'école doit-elle s'adapter à cette fatalité et renoncer à sa mission émancipatrice ? L'efficacité de l'éducation exclut-elle définitivement l'égalité ? Qualité de l'enseignement et égalité devant l'éducation sont-elles antinomiques?

L'éducation comparée doit permettre de mesurer l'acuité et l'ampleur de cet oxymore. À cet effet, les analyses comparatistes ${ }^{1}$ permettent de cerner ce qui constitue le « cœur» des inégalités scolaires à l'aide des indicateurs d'inégalité largement mis en œuvre dans les évaluations nationales et internationales ${ }^{2}$.

1. Colloque de la Revue internationale d'éducation de Sèvres, "Un seul monde, une seule école ? », 7-9 mars 2009, Centre international d'études pédagogiques, Sèvres. Communications consultables sur : www.ciep.fr/ries/ colloque-2009/contributions.php/atelier-B.php.

2. Education et formations: "Comparaisons internationales", $n^{\circ} 78$ (novembre 2008); "Mesurer les inégalités sociales de scolarisation : méthodes et résultats. Projections à l'horizon 2015 ", $n^{\circ} 74$ (avril 2007) ; " L'école réduitelle les inégalités sociales? ", $\mathrm{n}^{\circ} 66$ (juillet 2003).

Regards sur l'éducation 2009 : les indicateurs de l'OCDE. 
Sans cesse affinés, les critères autorisent, auprès d'une lecture brute des résultats et des classements, des corrélations plus fines et révélatrices des dysfonctionnements de l'École ${ }^{3}$.

\section{DISPARITÉS TERRITORIALES}

Parmi les facteurs générant les inégalités dans l'éducation, un ensemble important relève de données exogènes à l'école elle-même.

En premier lieu, la prégnance de la géographie montre qu'en dépit de l'unification théorique des systèmes éducatifs nationaux, les différenciations territoriales induisent des écarts dans les conditions de scolarisation. Une combinaison complexe de données historiques, culturelles et linguistiques, économiques et sociales, découpe partout des espaces différenciés. Sur un vaste territoire, ces écarts aboutissent même à des scolarisations disjointes. La Chine offre sur ce point des exemples qui autorisent, en retour, un regard renouvelé sur des situations plus proches (Xing 2009). Le différentiel entre régions très urbanisées à l'Est de la Chine et régions majoritairement rurales à l'Ouest entraîne des discordances éducatives profondes. Les indicateurs ${ }^{4}$ éclairent les inégalités qui, dans un contexte de développement économique comme celui que connaît actuellement la Chine, croissent sans que l'écart se réduise pour autant entre les régions. Cette problématique est renforcée par l'opposition villes-campagnes, l'offre scolaire en milieu rural étant particulièrement déficiente. En Europe, l'Espagne présente une série de ruptures Nord-Sud croisées avec une opposition ville-campagne qui reste forte, en dépit des mesures de compensation destinées aux zones rurales telle la politique de «Atención a la diversidad " (Faraco 2009). L'Allemagne connaît, avec des différences Nord-Sud, des contrastes marqués entre Länder de l'Ouest et ceux de l'ex-RDA à l'Est (Tarazona 2009).

Qui plus est, le développement économique génère des mouvements migratoires importants vers les villes. Les campagnes s'invitent en quelque sorte au cœur du système scolaire urbain qui peine à répondre à de nouveaux besoins éducatifs, retardant un peu plus l'intégration des migrants. On retrouve dans la plupart des pays ces données fondamentales dont l'immigration postcoloniale n'est qu'une autre face. De surcroît, on assiste à une série de micro-migrations internes, d'évitements stratégiques, de quartier à quartier, du secteur public au privé, voire au sein même de l'établissement. On aboutit à une hiérarchisation des lieux d'enseignement, des enseignants eux-mêmes, voire à un processus de ghettoïsation de l'école, souvent à résonance ethnique. On entre dans la « spirale de l'échec ». Ces mouvements browniens sont le fait des parents ambitieux qui, en connaisseurs des arcanes, contribuent à la déstabilisation du système et à plus de ségrégation sociale. Ils sont aussi le fait d'enseignants saisis par un désir de

3. Hanushek E. A., Wößmann L. (2006) : "Does educational tracking effect performance and inequality? Differencesin-differences evidence across countries”, Economic Journal 116, pp. 63-76.

4. Indicateur de Gini, indice de précarité global, grilles internationales d'équité, PIRLS, PISA, etc. 
fuite devant des situations scolaires dégradées. À la recherche de solutions, les politiques éducatives oscillent entre l'unification-planification gestionnaire "à la française » et les efforts de décentralisation poussés jusqu'au fédéralisme, comme en Espagne (Faraco 2009). Partout, ces disparités territoriales se traduisent par des variations locales et régionales pénalisantes pour le système éducatif et le respect du principe d'égalité.

\section{INÉGALITÉS SOCIALES}

Autre problématique exogène dont l'école doit s'accommoder : les caractéristiques sociales induisant des différenciations dans l'espérance de vie scolaire et dans la réussite des élèves. Il s'agit d'un facteur central pour élucider les processus générant les inégalités. Le lien entre disparités sociales et inégalités scolaires a varié au cours des dernières décennies. Après une longue période d'inertie, une dynamique lente et limitée a pu être discernée, dans les pays où le lien entre l'origine sociale et le diplôme a décliné. Ce lien ne doit donc pas être considéré comme intangible. Néanmoins, les différences de scolarisation entre les divers groupes sociaux persistent depuis des décennies en dépit des études menées principalement dès les années 1960 et les réformes qui les ont accompagnées. Ces «tendances lourdes » incitent à une mesure prédictive de la réussite entre élèves favorisés par l'origine sociale de leurs parents (capital culturel mobilisable, ressources investies dans l'éducation) versus élèves défavorisés (handicap culturel, pauvreté). Ces clivages transcendent les découpages territoriaux. Ainsi, sur le territoire relativement homogène scolairement parlant d'un pays " riche » comme la France ( $5^{\mathrm{e}} \mathrm{PIB}$ au monde), de forts écarts existent dès le début de la scolarité (école maternelle) et sont corrélés dans le primaire et dans les ordres suivants (Duru-Bellat Bydanova 2009). Ce schéma se retrouve presque partout avec une opposition entre pays du nord où la scolarisation est plus égalitaire et ceux du sud où l'échec scolaire lié à l'appartenance sociale semble la règle. La grande pauvreté aggrave ces différenciations de données quasi biologiques. À l'échelle du continent le plus pauvre du monde, l'Amérique latine, la situation inégalitaire prend des proportions qui défient la comparaison, avec des implications multiples en matière de santé, de lutte pour la survie et de sécurité face à la criminalité (Roche 2009).

\section{INSTITUTIONS, STRUCTURES ÉDUCATIVES}

Un autre ensemble de paramètres relève plus de la problématique propre à l'école. Que peut faire l'école pour remédier aux inégalités d'origine sociale ? Les systèmes éducatifs offrent-t-ils de réelles réponses ? L'école est-elle un lieu de compensation, de médiation, de remédiation ? Ou bien a-t-elle définitivement échoué à gommer les inégalités au prix d'un grand désenchantement idéologique et philosophique au regard de sa mission originelle? 
Ces paramètres aggravent quelquefois les données issues des inégalités sociales car il arrive que l'école soit plus inégalitaire encore que la société ellemême ${ }^{5}$. La démocratisation de l'enseignement reste la réponse majeure à ces questions et elle dépend de mesures institutionnelles. Les réformes mises en œuvre par le pouvoir politique ont un impact positif si elles sont prises dans une perspective globale. Ainsi, une politique de prévention et de santé publique a sans conteste un effet positif sur le rendement du système scolaire. Inversement, un affaiblissement de la médecine scolaire engendre rapidement un accroissement des inégalités. Une politique globale impliquant de nombreux acteurs, du type "politique de la ville », produit des résultats dans certains domaines de la scolarisation, apportant des réponses aux phénomènes d'absentéisme, de violence, de décrochage. De même, la discrimination positive est efficace si elle entre dans un processus continu de reconnaissance collective, échappant de cette manière à la stigmatisation, forme perverse de la ségrégation.

L'augmentation du ratio des dépenses consacrées à l'éducation dans les dépenses publiques devient significative lorsqu'elle s'inscrit dans une politique globale. Une augmentation trop basse du ratio peut être mise en relation avec des résultats médiocres : c'est actuellement le cas en France et en Allemagne qui se situent en deçà des pays de l'OCDE $^{6}$ et où les inégalités augmentent. Partout, on s'accommode d'une différence récurrente de traitement budgétaire entre le primaire et le secondaire. Par habitude ? Par paresse ? On sait pourtant que tout est joué avant la sortie du primaire, les résultats catastrophiques constatés dans maints pays le confirment.

Plus ambitieuses sont les réformes concernant les structures. Généralement, le débat porte sur école de base versus système à filières. Cette dernière structure a prouvé son inefficacité pédagogique en même temps que sa dangerosité en matière de cohésion sociale (Tarazona 2009). L'élitisme basé sur le mérite, prôné pendant des décennies comme la marque des systèmes éducatifs démocratiques et dont "l'élitisme républicain » est l'archétype en France, trouve ses limites dans un monde sans cesse plus diversifié et changeant qui exige à tout moment de nouvelles aptitudes, la maîtrise de nouvelles compétences, de nouveaux codes inconnus à l'École. Les aptitudes intellectuelles nouvelles ne passent plus par la seule définition académique symbolisée par le diplôme et l'idéologie méritocratique est suspectée de justifier les inégalités sociales dans le système scolaire et universitaire (Duru-Bellat 2009). Devant le maintien et l'accroissement des inégalités de départ et en l'absence de toute correction, l'idéal d'une éducation fondée sur la méritocratie s'effrite. Il faut un certain

5. Dubet, F. : « Les maux de l'école », Le Nouvel observateur n² 2338, 2 septembre 2009. 6. OCDE, Regards surl'éducation, septembre 2009. 
courage politique, comme en Allemagne, pour revenir sur le système des filières qui apparaît pourtant consubstantiel au système éducatif germanique (Tarazona 2009). Par ailleurs, si l'on considère que la rupture maintenue entre primaire et secondaire inférieur demeure un autre facteur d'inégalité sociale, il faudra un immense courage politique pour unifier la scolarité obligatoire en lissant les deux ordres actuellement séparés, au profit d'une structure comprehensive.

L'une des sources traditionnelles d'inégalité dans l'éducation, reste le double réseau public-privé en matière de scolarité obligatoire (Faraco 2009). Le réseau privé qui relève majoritairement des églises (l'Église catholique en Espagne pour 50 \%, en France pour 90 \%), permet aux parents qui le peuvent de choisir un établissement pour des raisons géographiques, pédagogiques ou, plus rarement, religieuses. Ces établissements payants, bien que recevant l'aide de l'État (selon un contrat en France, un concierto en Espagne), peuvent opérer une sélection «naturelle » en faveur des classes aisées. En ne recevant qu'une infime partie des enfants des catégories pauvres ou issus de l'immigration, alors que les enfants des milieux favorisés y sont sur-représentés, le réseau privé ne remplit pas de mission de service public (Toulemonde 2003). En Espagne, l'enseignement privé est même ressenti comme favorisant la fragmentation sociale de l'éducation obligatoire (Faraco 2009).

La formation des enseignants constitue un autre enjeu dans la réduction des inégalités scolaires. L'accès à la fonction enseignante révèle des inégalités sociales dont la "reproduction » des élites est l'un des processus. Certes une meilleure qualification universitaire des enseignants peut être le gage d'une élévation générale du niveau de la formation. En contrepartie, en superposant des exigences académiques de haut niveau aux exigences professionnelles du recrutement, on renforce le système de sélection en faveur d'un profil d'enseignant socialement de plus en plus étroit. De plus, on augmente la confusion entre compétences professionnelles et savoirs universitaires ${ }^{7}$. Cette évolution aboutit à écarter les catégories sociales modestes des métiers de l'enseignement. Rien d'étonnant à ce que le recrutement des enseignants à un niveau élevé rencontre, en France, plus de succès dans le réseau sélectif de l'enseignement privé ${ }^{8}$. Le danger est de voir émerger une école perçue comme l'image inversée de la société.

D'autres solutions sont mises en œuvre, associant plus étroitement les collectivités au fonctionnement de l'éducation en faisant de l'école un lieu ouvert à la société civile. Mais le recrutement à ce niveau encourt alors les risques du localisme et d'une soumission trop grande au bassin d'emploi.

7. Filâtre, D. : « Il faut oser le voile sur une tradition de concours... », www.EDUCPROS.fr, $1^{\text {er }}$ septembre 2009. 8. Lettre de d'éducation, $\mathrm{n}^{\circ} 639,7$ septembre 2009. 


\section{ACTEURS, PRATIQUES ÉdUCATIVES}

Le statut et le salaire des enseignants, déterminent leur place dans la société et le rôle qu'ils sont amenés à y jouer. Du niveau des salaires, qui restent très échelonnés parmi les pays de l'OCDE ${ }^{9}$, dépend la qualité du recrutement des enseignants, des gestionnaires et des cadres ; ce dernier point est fondamental tant la difficulté du métier nécessite le recours à des référents attentifs et compétents. Là encore, des mesures collectives et suivies obtiennent des résultats à long terme. La qualité des acteurs de l'enseignement, la pertinence de leurs stratégies sont déterminantes dans la perception et la prise en compte des inégalités scolaires. La conscientisation de l'enseignant à sa mission régulatrice devrait toujours être l'article premier du contrat passé avec la société. Or c'est à ce niveau que commence souvent une discrimination liée à l'appartenance sociale, discrimination que l'on dit, certes, inconsciente. En revanche, le soutien modulé prenant en compte les acquis antérieurs des élèves est peu répandu, à l'exception des pays du Nord où l'enseignement est fréquemment individualisé ${ }^{10}$.

L'effet « établissement » entre pour près de $50 \%$ dans les variances des évaluations. Son rôle modélisant influe sur la socialisation et l'exercice de la responsabilité de tous les acteurs. Les institutions locales, conseils, instances d'évaluation, d'orientation, de gestion, les règlements intérieurs sont-ils perçus comme des modèles de vie citoyenne, de justice ? Au delà du formalisme de la représentativité élective, a-t-on le courage d'assumer la part d'incertitude que comportent toute liberté, toute autonomie?

Enfin, on n'en finit pas de mesurer l'effet "parents " que certains systèmes éducatifs croient tenir à l'écart, mais qui en fait perpétue les inégalités entre élèves, en exigeant le maintien, souvent avec l'accord tacite des enseignants, de stratégies sélectives par simple référence au passé. Ainsi en va-t-il du redoublement qui pénalise irrémédiablement le parcours scolaire. Seule une politique scolaire cohérente peut s'opposer à ces attitudes rétrogrades et en souligner la nocivité.

\section{RÉPONSES GLOBALES, RÉPONSES LOCALES : QUELQUES PISTES}

Les inégalités en éducation constituent probablement la plus grande injustice que l'on puisse commettre envers de futurs citoyens. Pire, elles génèrent, un fort déficit de forces productives et créatives, une perte de capital

9. OCDE, Regards sur l'éducation, 2008.

10. Bouvier, Alain: "Les acquis des élèves ", Revue internationale d'éducation de Sèvres $n^{\circ}$ 43, décembre 2006, $17-22$. 
humain pour la société. Elles entravent fortement les processus d'intégration et de cohésion sociale. Il n'est certes pas du pouvoir de l'école de faire disparaître toutes les causes des inégalités face à l'éducation. Sur un plan local, au moins peut-elle limiter les effets de procédures ségrégatives, telle l'orientation scolaire et professionnelle trop souvent inscrite dans le déterminisme social. Les choix culturels des enseignants sont importants eux aussi. Négliger des aspects de la culture commune dans une perspective élitiste revient à amputer le patrimoine culturel d'apports qui y ont leur place naturelle. Auprès de l'impérative maîtrise des codes de base, c'est au contraire vers une transposition didactique des projets d'apprentissage que l'école doit se tourner (Roche 2009).

Puisqu'il est prouvé que l'éducation contribue directement à accroître la citoyenneté et l'engagement politique nécessaires à la démocratie ${ }^{11}$, l'école peut fournir à travers ses institutions et son propre fonctionnement un modèle qui ne sera pas oublié : celui de la démocratie fondée sur le contrat social et l'équité.

Les réponses plus globales relèvent de la prise de décision politique. On connaît la faible incidence des mesures sectorielles limitées à un seul aspect (les horaires, les programmes, les méthodes pédagogiques, les mesures de soutien scolaire...). D’une manière générale, trop de réformes de détail tuent la réforme. Leur multiplication entraîne la dérive vers un système éducatif fragmenté : c'est le cas en Espagne avec le développement des Autonomias jalouses de leurs prérogatives en matière d'éducation et dont les résultats sont très hétérogènes (Faraco 2009). On rencontre en Allemagne le même phénomène, avec des disparités persistantes entre les Länder qui privilégient la sélection et ceux qui mettent l'accent sur la performance individuelle (Tarazona 2009).

Les meilleurs résultats obtenus dans les évaluations internationales, sont souvent homothétiques de systèmes éducatifs plus proches du niveau local et autonomes dans leur fonctionnement (Pays-Bas, Danemark, Finlande), ce qui n'exclut pas un centre fort qui gouverne réellement en laissant la gestion se faire à la périphérie. Ces systèmes associent tous les partenaires de l'école selon une forme de gouvernance visible qui implique de réels pouvoirs locaux, des moyens financiers adéquats, une responsabilisation à tous les niveaux. Cela implique une redistribution complète des pouvoirs qui va à l'encontre des structures pyramidales et autoritaires qui ne produisent plus guère qu'une "illusion égalitaire " (Duru-Bellat Bydanova 2009). Cela suppose une école ouverte sur son environnement sociétal, un espace commun à la disposition des collectivités, un temps scolaire dilaté répondant aux besoins du corps social et un corps enseignant responsable, à haute reconnaissance salariale.

11. OCDE : Regards sur l'éducation, septembre 2009. 
La longue marche vers plus d'équité en éducation est loin d'être achevée. Mais les progrès accomplis ici et là, sont réels. Ils révèlent dans tous les pays une marge de manœuvre encore considérable. S'ils nécessitent une mobilisation des compétences de toutes les catégories professionnelles de l'enseignement, ils impliquent aussi une redéfinition politique des missions de l'éducation fondées sur un projet de société orienté vers plus de justice sociale et de bonheur.

\section{BIBLIOGRAPHIE}

DURU-BELLAT M., BYDANOVA L. (2009) : " L'éducation nationale : l'illusion égalitaire ? ». Revue internationale d'éducation de Sèvres. [http://www.ciep.fr/ries/colloque2009/atelier-C.php].

DURU-BELLAT M. (2009) : Le mérite contre la justice, Presses de Sciences Po.

GONZALEZ FARACO J.-C. : "Vieilles et nouvelles sources d'inégalités dans l'éducation espagnole ». Revue internationale d'éducation de Sèvres. [http://www.ciep.fr/ries/ colloque-2009/atelier-C.php].

ROCHE P. (2009) : «Pauvreté et inégalités scolaires en Amérique latine ». Revue internationale d'éducation de Sèvres. [http://www.ciep.fr/ries/colloque-2009/atelier-C.php].

TARAZONA M. (2009) : "Filières et disparités sociales et régionales dans le système scolaire allemand ». Revue internationale d'éducation de Sèvres. [http://www.ciep.fr/ ries/colloque-2009/atelier-C.php].

TOULEMONDE B. (2003) : in Le système éducatif en France, Documentation française.

XING K. (2009) : Revue internationale d'éducation de Sèvres. [http://www.ciep.fr/ries/ colloque-2009/atelier-C.php]. 